\title{
Observation of focusing effect in optical transition and diffraction radiation generated from a spherical target
}

\author{
L. G. Sukhikh, ${ }^{1}{ }^{*}$ A. S. Aryshev, ${ }^{2}$ P. V. Karataev, ${ }^{3}$ G. A. Naumenko, ${ }^{1}$ A. P. Potylitsyn, ${ }^{1}$ N. Terunuma, ${ }^{2}$ and J. Urakawa ${ }^{2}$ \\ ${ }^{1}$ Tomsk Polytechnic University, Lenin Avenue 30, Tomsk, 634050, Russia \\ ${ }^{2}$ KEK: High Energy Accelerator Research Organization, 1-1 Oho, 305-0801 Tsukuba-shi, Ibaraki-ken, Japan \\ ${ }^{3}$ John Adams Institute at Royal Holloway, University of London, Egham, Surrey, TW20 OEX, United Kingdom
}

(Received 22 February 2008; published 20 July 2009)

For the first time the focusing effect in optical transition and diffraction radiation generated by $1.28 \mathrm{GeV}$ electrons in a tilted spherical target has been observed experimentally. A comparison of detected as well as simulated radiation spatial distributions produced by a flat and a spherical target has been made. It is shown that the application of such targets has allowed us to increase the radiation spectralspatial density at the target focus without applying any additional focusing devices.

\section{INTRODUCTION}

Transition (TR) and diffraction (DR) radiation phenomena have a very broad spectrum of applications. For example, they are widely used for electron beam diagnostics. The TR appearing when a charged particle crosses a vacuum-metal interface is implemented for transverse beam profile measurements because it is possible to achieve a high resolution and perform a shot-by-shot monitoring of beam parameters. The processes of TR and DR generation are very similar [1]. However, using DR one can develop an almost noninvasive diagnostics method. In the experiment [2] the transverse beam size was measured using optical DR (ODR) with an accuracy of the order of $14 \mu \mathrm{m}$. It is also possible to measure a longitudinal beam profile through the analysis of coherent TR or DR spectral characteristics [3]. Recently, we proposed to use focused coherent DR for soft $x$-ray generation via the inverse Thompson scattering process [4].

In applications mentioned above, there is one serious problem related to the "prewave zone" effect for backward TR and DR emitted at an angle much larger than $1 / \gamma$, where $\gamma$ is the charged particle Lorentz factor. Sometimes this effect is called "near field," but this term seems to be inappropriate. Indeed, the near-field zone is concerned with the static field that is significant only at distances comparable with the radiation wavelength $\lambda$ [5]. The prewave zone effect becomes significant when the distance between a target and a detector is shorter than $\gamma^{2} \lambda \gg \lambda$ [6]. In the prewave zone, the field is not static but is instead a radiation one. The physical origin of the prewave zone effect is the influence of the finite radiation region (which is proportional to $\gamma \lambda$ ) on the detected radiation characteristics. The "formation length" for forward transition radiation corresponds to the distance at which the fields of

\footnotetext{
*Sukhikh@interact.phtd.tpu.ru
}

radiation and the particle propagating almost in the same direction do not interact with each other and has the same criterion of $\left(\gamma^{2} \lambda\right)$. But the physical origin is quite different in the case of the prewave zone. The formation length criterion may be written in the following form $[7,8]$ :

$$
L_{f}=\frac{\beta \lambda}{2(1-\beta \cos \theta)} .
$$

Here $\beta$ is the electron velocity in units of the speed of light, $\theta$ is the radiation angle. For forward TR the angle $\theta$ is close to zero in the ultrarelativistic case and the formation length criterion transfers to the well-known $L_{f}=\gamma^{2} \lambda$. For the backward TR the angle $\theta$ is close to the mirror reflection direction (i.e. $\pi / 2$ for our experimental case). One may see that the formation length in this case is comparable to the radiation wavelength $\lambda \ll \gamma^{2} \lambda$.

The prewave zone effect results in a TR (DR) spectralspatial density distortion that seriously reduces the efficiency of TR (DR) practical applications.

The prevalent method for TR (DR) focusing is to use external focusing components such as lenses and/or paraboloidal mirrors $[9,10]$. However, the use of such components might be complicated or even impossible in some applications such as soft $x$-ray generation. In $[9,11,12]$ the authors proposed to use paraboloidal targets in order to focus the TR (DR) without complicated external optical components. In [11] the authors performed simulations using the surface current model, while in $[9,12]$ the authors used the Huygens model. The latter one is based on the replacement of the real electron field by the field of virtual photons known as the Weizsacker-Williams method (applied to the DR problem in [13]) and the Huygens reflection of these photons from the TR (DR) target. It is well known that the Huygens model is approximate and there is a strict region for kinematic variables as has been recently shown in the paper [14]. Good calculation accuracy may be achieved only for almost normal incidence. It was shown 


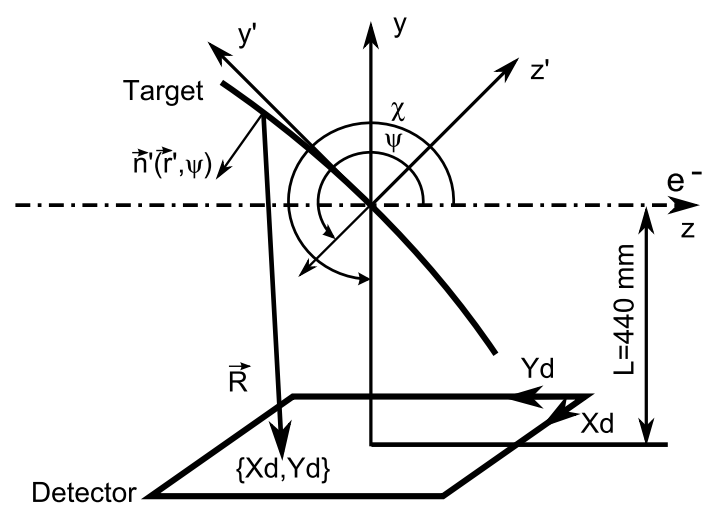

FIG. 1. The simulation scheme and some definitions.

that the surface current model is limited to the same condition [15]. Furthermore, up to now there is no clear argument about the possibility of using these models for tilted concave TR (DR) targets.

Fortunately, in the recent papers $[14,15]$ the authors independently proposed the vector model of TR (DR) generation allowing the TR (DR) characteristics for targets tilted with respect to the electron trajectory to be obtained.

The TR (DR) electric field $\left(\boldsymbol{E}_{\boldsymbol{d}}\right)$ with wavelength $\lambda$ in a detecting point with coordinates $\boldsymbol{R}_{\boldsymbol{d}}$ (see Fig. 1) may be expressed following the papers $[14,15]$ as

$$
\boldsymbol{E}_{d}\left(\boldsymbol{R}_{\boldsymbol{d}}, \lambda\right)=\frac{1}{2 \pi} \int\left[\boldsymbol{n} \times \boldsymbol{E}_{\boldsymbol{e}}\right] \times \nabla G d S .
$$

Here $\boldsymbol{n}$ is the unit normal to the target surface, $\boldsymbol{E}_{\boldsymbol{e}}(\boldsymbol{\rho}, z, \lambda)=\frac{2 e}{\gamma \lambda c} e^{i k z} \frac{\boldsymbol{\rho}}{\rho} K_{1}\left(\frac{2 \pi}{\gamma \lambda} \rho\right)$ is the electron field Fourier transform taking into account the ultrarelativistic limit, $\boldsymbol{\rho}=\{x, y\}, K_{1}$ is the modified Bessel function of the second kind, $\nabla G$ is the Green function gradient, $G=\frac{e^{i k R}}{R}$, $k=2 \pi / \lambda, R=|\boldsymbol{R}|=\left|\boldsymbol{r}-\boldsymbol{R}_{\boldsymbol{d}}\right|$ is the distance between an arbitrary point on the target surface and one on the detector surface. The integration is performed over the target sur-

face $S$. Taking into account the surface element $d S=$ $\frac{\sqrt{x^{2}+y^{2}+4 f^{2}}}{2 f} d x d y$, where $f$ is the target focal distance, one may easily simulate the spectral-spatial distribution of radiation generated in the paraboloidal target in the following way:

$$
\frac{d^{2} W}{\hbar d \omega d \Omega}=\frac{c}{\hbar} L^{2}\left|\boldsymbol{E}_{\boldsymbol{d}}\right|^{2}
$$

Here $L$ is the distance between the centers of the target and the detector.

It is well known that if the size of the TR (DR) spot on the target surface, which is of order $\gamma \lambda$, is much smaller than its focal distance, a paraboloidal surface may be approximated by a spherical one. For a spherical target the focal distance $f$ is equal to half the sphere curvature radius. For a flat target $f$ tends to infinity.

The model predicts that the spatial distributions of the optical TR (OTR) and the ODR from a tilted paraboloidal or a spherical target in the focus should be very close to the far-field ones, i.e., such targets focus the OTR (ODR) distributions compared to the flat ones if $f \ll \gamma^{2} \lambda$. Also there should be a difference in spatial distributions from the tilted and nontilted spherical targets. These simulation results are shown below together with the experimental results.

\section{EXPERIMENTAL SETUP}

In order to test the theoretical predictions, we performed a proof-of-principle experiment at the KEK-ATF (Accelerator Test Facility) to observe the focusing effect in the OTR and the ODR phenomena. Some preliminary results were represented in [16]. The experimental setup is shown in Fig. 2. It was installed in the diagnostics section of the KEK-ATF extraction line [2]. The extracted beam parameters in the vicinity of the target during the experiment are listed in Table I.

The vacuum chamber had a target stage actuator with a linear movement accuracy better than $0.5 \mu \mathrm{m}$. The target holder mounted on the stage at $45^{\circ}$ angle with respect to the beam line was designed to carry two target samples. A $10 \mu \mathrm{m}$ thick tungsten wire used to measure the vertical beam size (see Table I) was installed in the target chamber. There was no possibility to measure the horizontal beam

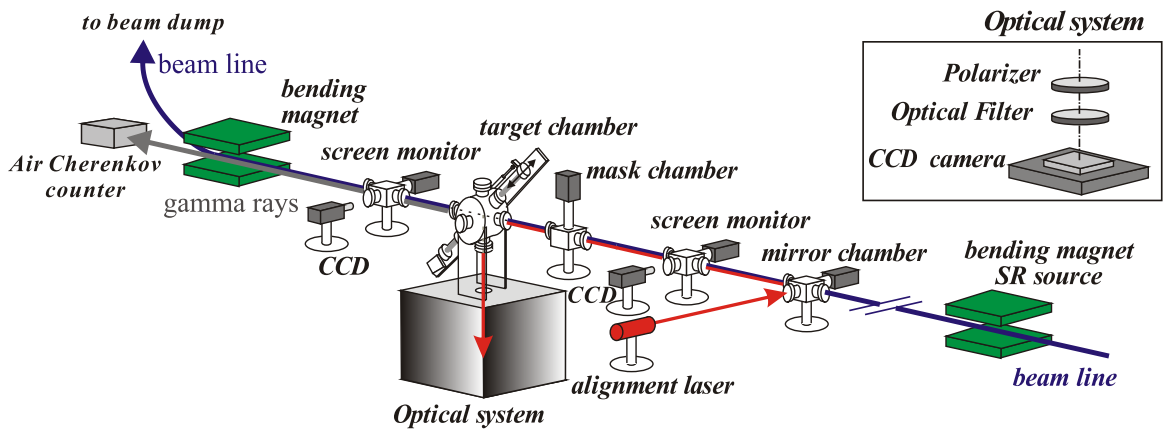

FIG. 2. (Color) Experimental setup. 
TABLE I. Beam parameters near the target.

\begin{tabular}{lc}
\hline \hline Energy & $1.28 \mathrm{GeV}(\gamma=2500)$ \\
Bunch population & $0.9 \times 10^{10} \pm 15 \%$ \\
Bunch repetition & $1.56 \mathrm{~Hz}$ \\
Vertical beam size & $\sigma_{\perp}=29.35 \pm 1.1 \mu \mathrm{m}$ \\
Horizontal beam size & $\sigma_{\|}<150 \mu \mathrm{m}$ \\
\hline \hline
\end{tabular}

size near the target location. The horizontal beam size mentioned in Table I was predicted by the SAD code [17].

The flat target was a $300 \mu \mathrm{m}$ thick gold-coated silicon wafer with $(7 \times 9) \mathrm{mm}^{2}$ dimensions [2]. A commercially available spherical target was an aluminized glass segment with transverse dimensions of $(15 \times 7.5) \mathrm{mm}^{2}$ and $500 \mathrm{~mm}$ focus. The targets allowed us to measure both OTR and ODR. The position of the target with respect to the electron beam is very important in this case. Measuring OTR, one must be sure that there is no edge effect. Measuring ODR one should precisely know the impact parameter (the shortest distance between the target edge and the electron trajectory). An air Cherenkov counter set up downstream was used to measure the bremsstrahlung photons generated when the electron beam directly interacted with the target. The dependence of the detector response versus target position (Fig. 3) allowed us to define the impact parameter [18]. The air Cherenkov signal dependence for a single electron has the form shown by the dashed line in Fig. 3. The point where this linear fit crossed the abscissa is assumed to be the point of zero impact parameter. Because of the nearly Gaussian distribution of the electrons in the vertical direction at the point of zero impact parameter, half of the beam interacted with the target directly. The solid curve in Fig. 3 shows the single electron dependence convoluted with a Gaussian distribution with $\sigma_{y}=29.35 \mu \mathrm{m}$ (see Table I).

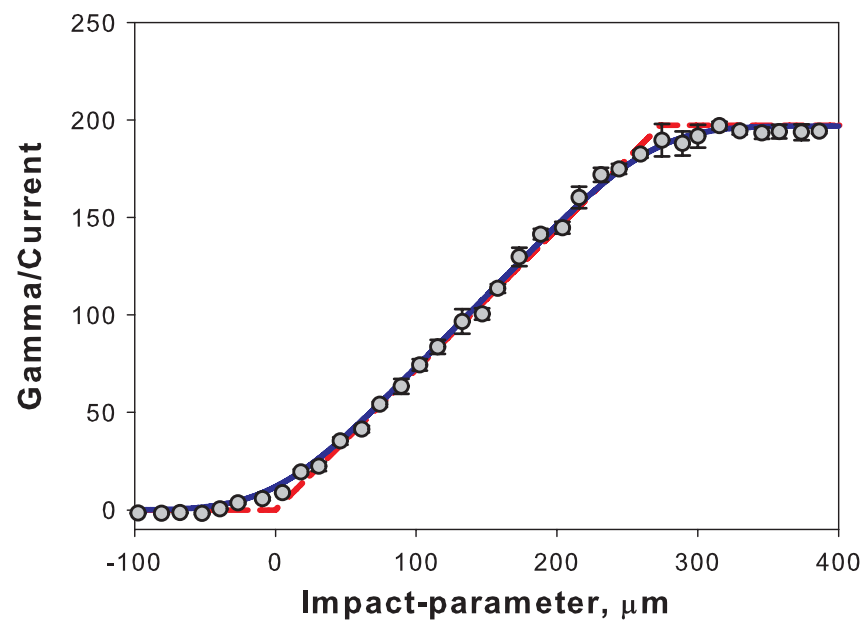

FIG. 3. (Color) The dependence of air Cherenkov counter signal vs impact parameter (points); single electron approach (dashed red line); Gaussian bunch $\left(\sigma_{y}=29.35 \mu \mathrm{m}\right)$ approach (solid blue line).

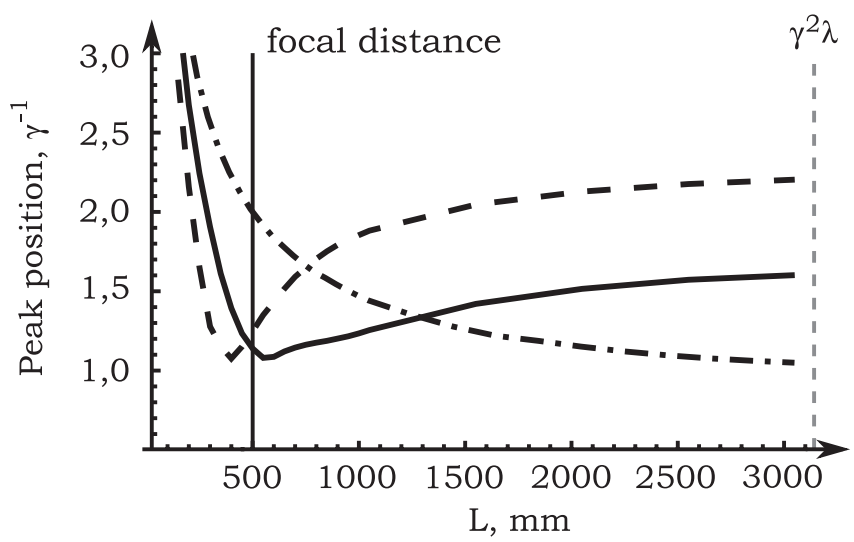

FIG. 4. The dependence of both polarization OTR peak position vs target-detector distance for the tilted parabolic target with $f=500 \mathrm{~mm}$ (solid curve- $X d$ direction; dashed curve- $Y d$ direction) and the same dependence for the flat target (dashand-dotted curve).

The ATF extraction line contains many different magnetic devices, which might lead to the appearance of synchrotron radiation (SR) background. To avoid the SR contribution a mask has been installed $0.3 \mathrm{~m}$ upstream of the target to cut the SR off. The mask itself was a ceramic plate with a $(1 \times 2) \mathrm{mm}^{2}$ hole in the center of it [19]. The mask hole was centered on the beam path and there were no effects on the beam due to charge buildup on the plate.

The optical system consisted of a polarizer, an optical filter, and a highly sensitive cooled CCD camera (Alta E4000). The pixel size of the camera is equal to $(7.4 \times$ 7.4) $\mu \mathrm{m}^{2}$. The high quantum efficiency $(55 \%$ for $\lambda=$ $550 \mathrm{~nm}$ ) along with high bunch population allowed us to carry out single-shot measurements. All results shown below were obtained using single-shot measurements. The background and camera dark current were measured while the targets were removed and averaged over ten shots. The detector was installed at a distance of $L=$ $440 \mathrm{~mm}$, which corresponds to $0.14 \gamma^{2} \lambda$ (an extreme prewave zone) for $\lambda=500 \mathrm{~nm}$. Unfortunately, it was not possible to change the distance between the target and the detector because of technical limitations. Figure 4 shows the simulated dependence of the OTR peak position for both polarizations versus target-detector distance for a tilted parabolic target with $f=500 \mathrm{~mm}$ (solid curve $X d$ direction, dashed curve $Y d$ direction) and the same dependence for a flat target (dash-and-dotted curve). One can see that for the flat target the maximum converges to $\gamma^{-1}$ but for the tilted parabolic target the peak position is minimal at a different point near the focal one. Figure 4 explains why the choice of the target focal distance is not equal to target-detector distance. Because of the target tilt the image becomes astigmatic which means that it is impossible to obtain an ideally focused spatial distribution coinciding with the well-known far-field one. Thus, the "best" focusing is at the distance $L \simeq 440 \mathrm{~mm}$ for the target focal distance of $f=500 \mathrm{~mm}$. 


\section{EXPERIMENTAL RESULTS AND DISCUSSION}

During the first part of the experiment the vertical polarization component $\left(E_{\perp}^{d}\right)$-the component along the $Y d$ direction in our definitions of the OTR without the optical filters-was measured. In order to accommodate the model to the experiment, the influence of the joint action of the CCD camera spectral efficiency $F(\omega)$ and the Fresnel reflection coefficients $R_{\perp}$ (see, for instance, [19]) of the target materials had to be included. In order to take into account the transverse bunch dimensions, one should convolute the radiation intensity with the electron bunch profile $\phi\left(y, \sigma_{\perp}\right)$, which was assumed to be a Gaussian with $\sigma_{\perp}=29.35 \mu \mathrm{m}$ (see Table I):

$$
\frac{d W_{\perp}}{d \Omega}=\mathrm{const} \iint F(\omega)\left|R_{\perp}(\omega)\right|^{2}\left|E_{\perp}^{d}\right|^{2} \phi\left(y, \sigma_{\perp}\right) d \omega d y .
$$

The convolution with the horizontal beam profile was not carried out because for the vertical polarization component the horizontal beam size effect is insignificant.

All further simulations in the first part of the experiment were carried out using Eq. (3). The numerical integration was performed using a Monte Carlo technique. In all experimental figures gray circles are used for spherical target experimental distributions, white squares-flat target experimental distributions, solid lines-spherical target simulations, and dashed lines-flat target simulations.

In Fig. 5 one can see that the OTR spatial distribution from the spherical target is narrower than that from the flat one by a factor of 2 as the theory predicted. The interpeak distance of the OTR distribution from the spherical target is $2.46 \pm 0.02 \gamma^{-1}$, and from the flat one $-4.30 \pm 0.03 \gamma^{-1}$. For the simulated distributions these values are $2.28 \gamma^{-1}$

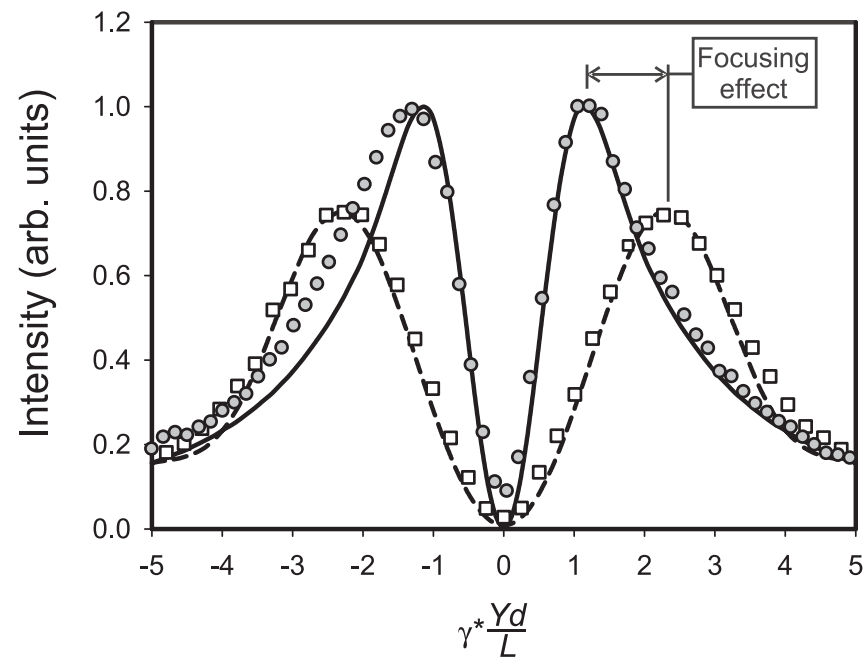

FIG. 5. The OTR vertical polarization component spatial distributions along the $Y d$ direction $(X d=0)$. Gray dots-spherical target experiment; white squares-flat target experiment; solid line_-spherical target theory; dashed line-flat target theory.

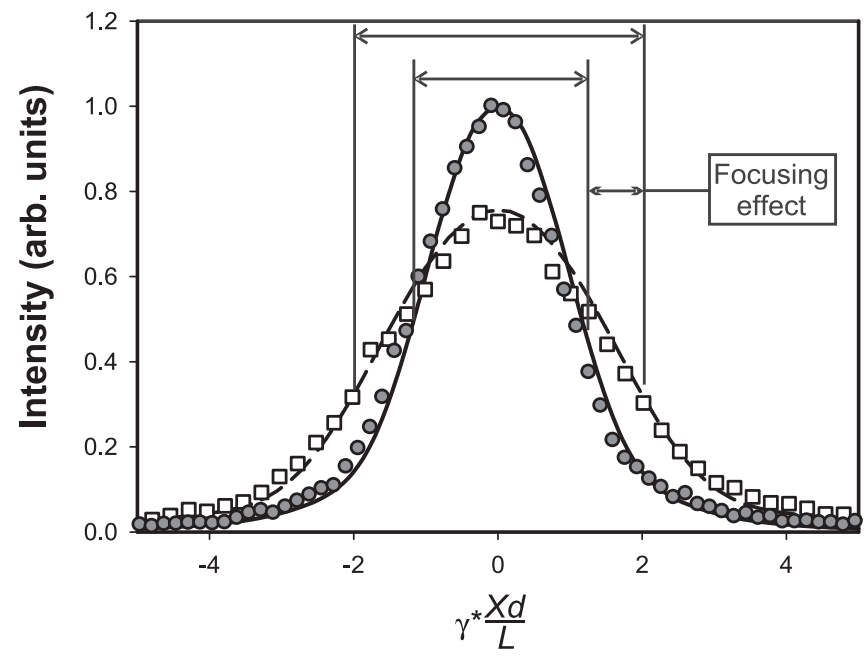

FIG. 6. The OTR vertical polarization component spatial distributions along the $X d$ direction $(Y d=\max )$. Gray dotsspherical target experiment; white squares-flat target experiment; solid line_-spherical target theory; dashed line-flat target theory.

and $4.4 \gamma^{-1}$, respectively. Thus, one can see that the measured values agree with the simulations very well. One should note that the relative intensity also agrees well with the theory. The spatial distribution from the spherical target in the prewave zone is very similar to the far-field zone distribution with the well-known interpeak distance of $2 \gamma^{-1}$. A small deviation from the far-field distribution comes from the geometry of a tilted target. A little difference between the theoretical distribution and the measured data for the spherical target may be caused by a small SR contribution that may also be focused by the spherical surface.

Figure 6 shows the simulated and measured distributions in the $X d$ direction for the peak $(Y d=\max )$. In order not to overload the figure only one peak is shown. Table II shows the full widths at half maxima (FWHM) for both peaks for the estimated and measured distributions. In Table II and Fig. 6 one may see good consistency between the theoretical and the experimental results.

It is well known that the ODR spatial distribution in the far-field is symmetrical [18]. In the prewave zone the spatial distribution becomes skewed in one direction $(Y d$ in our definitions). Figure 7 shows the experimental and theoretical (simulated for the incidence on the tilted target

TABLE II. Estimated and experimental values of the FWHM of OTR angular distribution peaks.

\begin{tabular}{lccc}
\hline \hline Target & Theory, FWHM, $\frac{\gamma X d}{L}$ & \multicolumn{2}{c}{ Experiment, } \\
& & $Y d<0$ & $Y d>0$ \\
\hline Spherical & 2.28 & $2.17 \pm 0.062$ & $2.25 \pm 0.050$ \\
Flat & 3.54 & $3.97 \pm 0.100$ & $3.5 \pm 0.088$ \\
\hline \hline
\end{tabular}




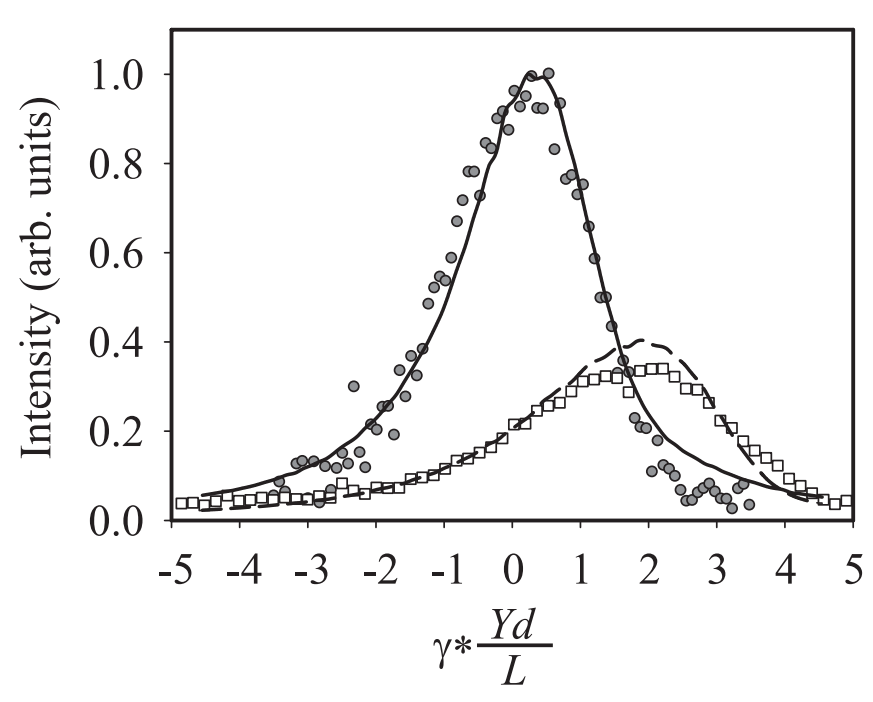

FIG. 7. The ODR vertical polarization component spatial distributions along the $Y d$ direction $(X d=0)$. Gray dots-spherical target experiment; white squares-flat target experiment; solid line-spherical target theory; dashed line-flat target theory. The impact parameter is equal to $h=50 \mu \mathrm{m}$.

surface $\psi=5 \pi / 4, \chi=3 \pi / 2$, see Fig. 1) ODR spatial distributions from both targets. In order to take into account the asymmetry, the following fit function was introduced:

$$
f(x)=a \exp \left[-\frac{1}{2}\left(\frac{x-x_{0}}{b\left(1-M_{3} \operatorname{sign}\left(x-x_{0}\right)\right)}\right)^{c}\right] .
$$

Here $a, b, c$, and $x_{0}$ are the fit parameters and $M_{3}$ is the asymmetry coefficient. The ODR spatial distributions were fitted by this function in order to obtain the asymmetry coefficients and FWHM. The asymmetry coefficients for the experimental ODR spatial distribution from the spherical and flat targets are equal to $0.186 \pm 0.028$ and $0.305 \pm$ 0.023 , respectively. The corresponding theoretical values are 0.170 and 0.352 , respectively. One may say that the mentioned asymmetry was caused by the difference between the focal distance $f$ and the distance from the target to the detector $L$. In order to show that the target tilt angle also should be taken into account, we simulate the normal incidence case $(\psi=\chi=\pi)$. For the normal incidence case the asymmetry coefficients for the spherical and flat targets are 0.097 and 0.331 , respectively. As one might expect there is no serious difference in the ODR spatial distributions from the tilted and nontilted flat target. But there is a difference (almost factor 2) between the ODR spatial distributions from tilted and nontilted spherical targets.

The main characteristic of the ODR is the dependence of the ODR spatial distribution FWHM in the horizontal direction $\left(\frac{\gamma X d}{L}\right)$ vs the impact parameter [18]. This dependence is shown in Fig. 8. In Fig. 8 one may clearly see that the ODR distribution from the spherical target is narrower

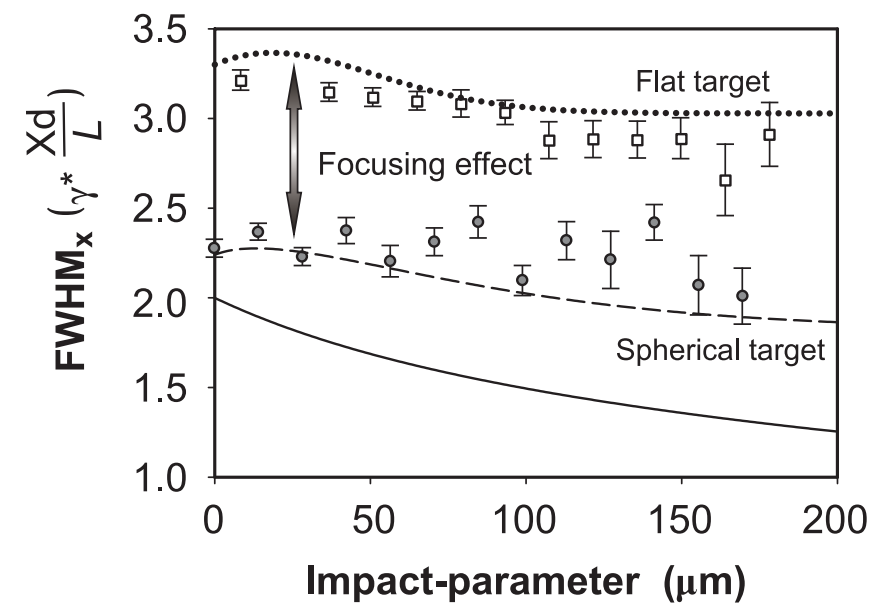

FIG. 8. The ODR vertical polarization component FWHM vs impact parameter. Gray dots-spherical target experiment; white squares-flat target experiment; solid line-spherical target theory; dashed line-flat target theory; dotted line-single electron far-field approach.

than from the flat one by a factor of 1.5. Because of the exponential dependence of the ODR photon yield as a function of the impact parameter for lower ODR intensities, the relative statistical error is larger. The errors are obtained by fitting the measured distributions to the function (4). Nonexponential dependence in the region of small impact parameters was caused by a nonzero vertical beam size.

In the next part of the experiment the total OTR distributions with an optical filter $\lambda=609 \mathrm{~nm}$ were measured.

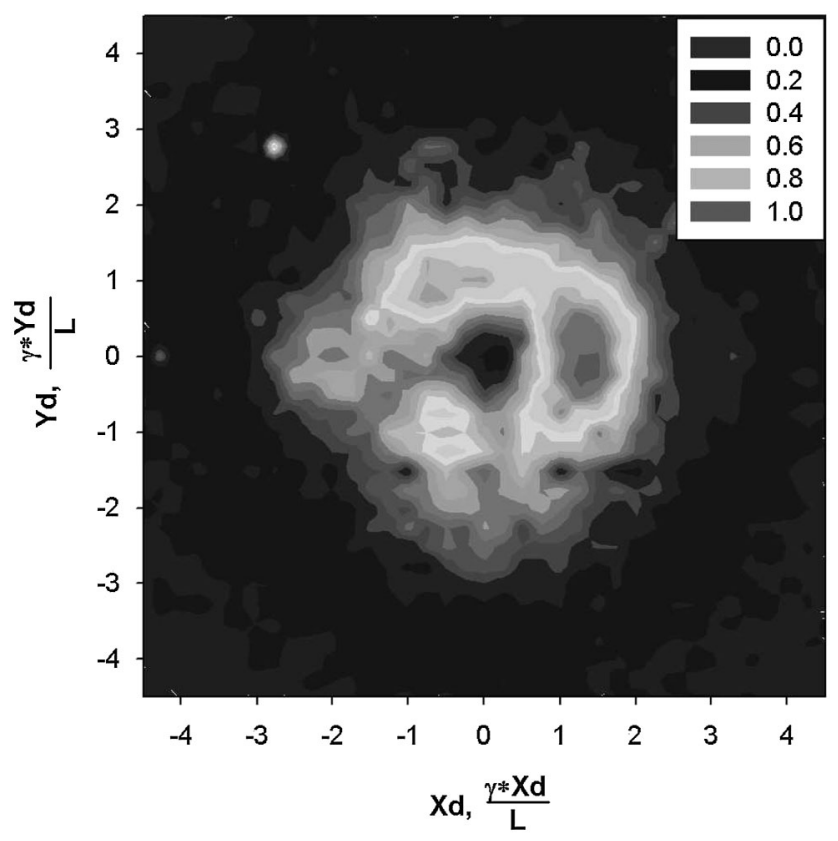

FIG. 9. Both polarization components of OTR spatial distribution for a $609 \pm 28 \mathrm{~nm}$ filter from a spherical target. 


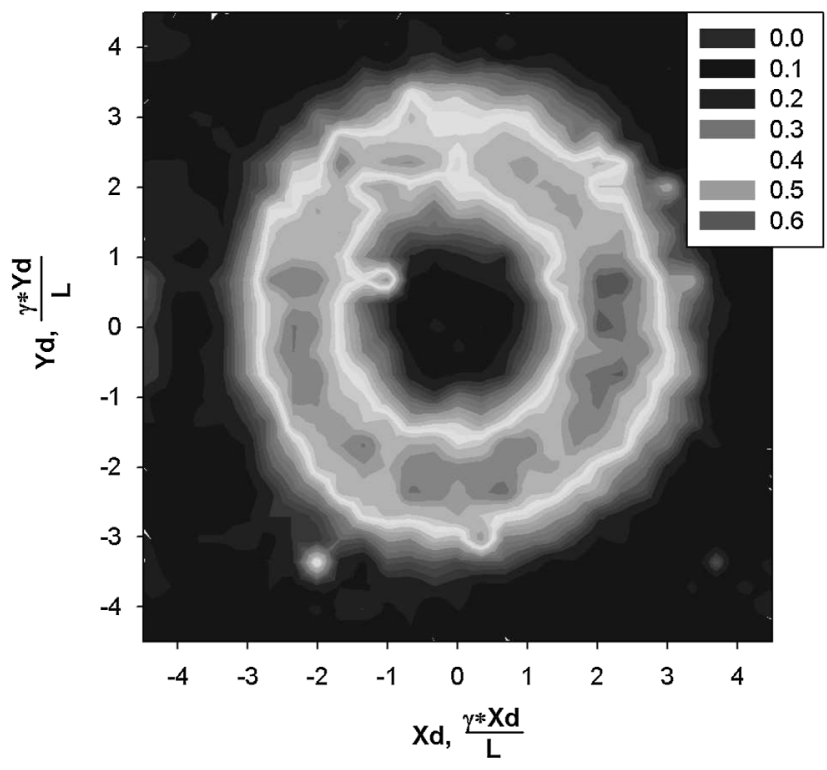

FIG. 10. Both polarization components of OTR spatial distribution for a $609 \pm 28 \mathrm{~nm}$ filter from a flat target.

The filter bandwidth at $10 \%$ level is equal to $56 \mathrm{~nm}$. The simulations in this part were made using Eq. (2). Figures 9 and 10 show the experimentally measured 3D distributions of the OTR measured from spherical and flat targets. The experimentally measured 3D OTR distributions were recalculated in polar coordinates. Figure 11 shows the OTR radial distribution measured from both spherical and flat targets $\left(R d=\sqrt{X d^{2}+Y d^{2}}\right)$. The distributions were averaged over the azimuthal angle. The theoretical simulations were performed using the model in the same manner. In Fig. 11 one may clearly see that the theoretical predictions and experimental data are qualitatively in agreement. A

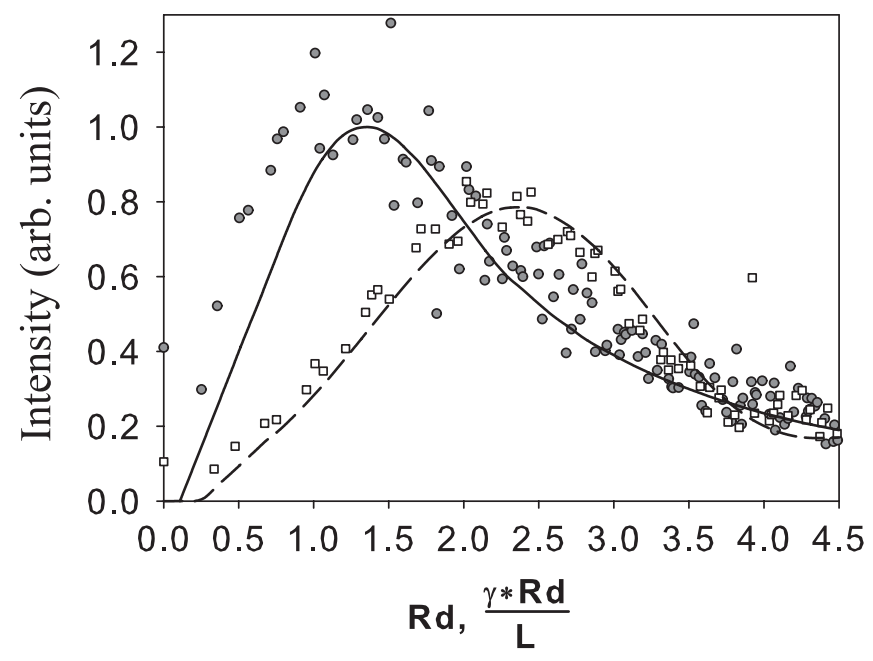

FIG. 11. The radial distribution of the total OTR intensities. Gray dots-spherical target experiment; white squares-flat target experiment; solid line-spherical target theory; dashed line-flat target theory. disagreement may be explained by the influence of a SR background that is larger in the case of both polarization components.

\section{CONCLUSION}

In this paper we have carried out single-shot measurements of OTR and ODR generated from a spherical target in the prewave zone. We have compared these distributions with those generated from a flat target. The flat target distributions are distorted because of the prewave zone effect. The OTR cone is broader than the far-field one by a factor of 2.2 and the ODR distribution is skewed. The spherical target distributions are very close to the wellknown far-field distributions in spite of the prewave zone effect, i.e., they are focused without the use of any additional external focusing devices.

All distributions have been observed with good reproducibility and are in good agreement with the theoretical predictions carried out using the vector TR (DR) model $[14,15]$. Good agreement was observed for both the spatial distributions and for the relative intensities.

We have shown that the concave target tilt seriously affects the TR (DR) distributions causing some distortions and astigmatism that do not allow distributions coinciding with the far-field ones to be obtained. The fact that the tilted spherical targets cause some differences in the OTR and ODR spatial distributions should be taken into account if one uses such targets. It seems that this problem may be solved using astigmatic targets that are not spherical but instead ellipsoidal ones with a proper relation between the focal distances.

The results allow us to hope that the concave TR (DR) target application can be widely adopted in different beam diagnostics tools. Concave targets might decrease the focus depth effect (i.e. image dilution) in the OTR imaging beam profile monitors placed even in an extreme prewave zone. Also we hope that using such focusing targets in applications of stimulated TR and DR generation increases the stimulated radiation yield. By using concave targets in an experiment like [20], one might significantly increase the spectral-spatial density of the radiation.

\section{ACKNOWLEDGMENTS}

We would like to acknowledge Professor Grahame Blair for his useful suggestions and criticism during the manuscript preparation.

[1] A. P. Potylitsyn, Nucl. Instrum. Methods Phys. Res., Sect. B 145, 169 (1998).

[2] P. Karataev, S. Araki, R. Hamatsu, H. Hayano, T. Muto, G. Naumenko, A. Potylitsyn, N. Terunuma, and J. Urakawa, Phys. Rev. Lett. 93, 244802 (2004). 
[3] Y. Shibata, T. Takahashi, T. Kanai, K. Ishi, M. Ikezawa, J. Ohkuma, S. Okuda, and T. Okada, Phys. Rev. E 50, 1479, (1994).

[4] A.P. Potylitsyn, L. G. Sukhikh, A.S. Kostousov, J. Urakawa, A. Aryshev, S. Boogert, and P. V. Karataev, in Proceedings of RuPAC 2006, Novosibirsk, Russia, 2006, edited by JACoW, http://cern.ch/AccelConf/r06/PAPERS/ MONO07.PDF.

[5] L. D. Landau and E. M. Lifshiz, The Classical Theory of Fields (Pergamon, Oxford, 1962), 2nd ed.

[6] V. A. Verzilov, Phys. Lett. A 273, 135 (2000).

[7] B. M. Bolotovsky, Trudy Fiz. Inst. Akad. Nauk USSR 140, 95 (1982) (in Russian).

[8] V.L. Ginzburg and V. N. Tsytovich, Transition Radiation and Transition Scattering (Adam Hilger, Bristol, 1990).

[9] P. V. Karataev, Phys. Lett. A 345, 428 (2005).

[10] B. N. Kalinin, G. A. Naumenko, A. P. Potylitsyn, G. A. Saruev, L. G. Sukhikh, and V. A. Cha, Pis'ma Zh. Eksp. Teor. Fiz. 84, 136 (2006) [JETP Lett. 83, 110 (2006)].

[11] M. I. Ryazanov and I. S. Tilinin, Sov. Phys. JETP 44, 1092 (1976).

[12] A. P. Potylitsyn and R. O. Rezaev, Nucl. Instrum. Methods Phys. Res., Sect. B 252, 44 (2006).
[13] M.L. Ter-Mikaelyan, High Energy Electromagnetic Processes in Condensed Media (Wiley-Interscience, New York, 1972).

[14] A. G. Shkvarunets and R. B. Fiorito, Phys. Rev. ST Accel. Beams 11, 012801 (2008).

[15] D. V. Karlovets and A. P. Potylitsyn, Zh. Eksp. Teor. Fiz. 134, 887 (2008) [JETP 107, 755 (2008)].

[16] L. G. Sukhikh, G. A. Naumenko, A.P. Potylitsyn, J. Urakawa, A.S. Aryshev, S. Boogert, and P. V. Karataev, in Proceedings of DIPAC 2007, Venice (Mestre), Italy, 2007, edited by Ivan Andrian, p. 259.

[17] SAD, Strategic Accelerator Design, http://acc-physics. kek.jp/SAD/sad.html.

[18] T. Muto, S. Araki, R. Hamatsu, H. Hayano, T. Hirose, P. Karataev, G. Naumenko, A. Potylitsyn, and J. Urakawa, Phys. Rev. Lett. 90, 104801 (2003).

[19] P. Karataev, Ph.D. thesis, Tokyo Metropolitan University, 2004.

[20] Y. Shibata, K. Ishi, T. Takahashi, T. Matsuyama, F. Hinode, and Y. Kondo, Nucl. Instrum. Methods Phys. Res., Sect. A 528, 162 (2004). 